\title{
- Original Article. \\ The cultivation and identification of tumor stem cells from neuroblastoma derived tumor spheres
}

\author{
Qiu-Xia Liu', Jing-Yan Tang' ${ }^{1}$, Jiao-Yang Cai', Min-Zhi Yin², Ben-Shang Li ${ }^{1}$ \\ ${ }^{1}$ Department of Hematology/Oncology, Shanghai Children's Medical Center, Shanghai Jiaotong University School of Medicine, Shanghai \\ 200127, P. R. China; ${ }^{2}$ Department of Pathology, Shanghai Children's Medical Center, Shanghai Jiaotong University School of Medicine, \\ Shanghai 200127, P. R. China
}

\begin{abstract}
[Abstract] Background and Objective: Since the proposal of the tumor stem cell hypothesis, considerable interest has been devoted to the isolation and purification of tumor stem cells. Tumor stem cell enrichment from primary tumor derived cell spheres has been demonstrated in specific, serum-free media. This goal of this study is to establish a method of cultivating floating tumor spheres from neuroblastoma cells and to confirm that neuroblastoma spheres are rich in tumor stem cells. Methods: Bone marrow aspirates were obtained from pediatric patients diagnosed with stage IV neuroblastoma. Primary tumor cells were isolated and cultivated in serum-free, stem cell-selective medium. Single sphereforming cells were cultivated under serum-free conditions; their cloning efficiency and monoclonal tumor sphere formation rates were calculated. The expression of stem cell marker genes Oct-4 and Bmi-1 was detected by RT-PCR in sphereforming cells and parental neurolastoma cells. Sphere-forming cells were injected into the armpit of nude mice with subsequent assessment for tumor growth. Sphere-forming cells were cultivated in differentiation medium containing $5 \mu \mathrm{mol} / \mathrm{L}$ 13-cis retinoic acid; changes in cell morphology were observed. Results: Neuroblastoma cells formed non-adherent neurospheres under serum-free, stem cell-selective conditions after a period of 4 to 6 days. A single cell dissociated from a neurosphere could reform a monoclonal sphere; cloning efficiency and monoclonal sphere formation rates were $55.3 \%$ and $26.3 \%$, respectively. RT-PCR results revealed heightened tumor sphere expression of Oct-4 and Bmi-1 as compared with parental tumor cells. Fourteen days after injection of $10^{4}$ sphere-forming cells into nude mice, a neuroblastoma xenograft formed. Treatment of sphere-forming cells with 13-cis retinoic acid induced a gradual differentiation to neuronal cell morphology. Conclusions: Neuroblastoma derived tumor spheres enrich tumor stem cells and the cultivation of primary neuroblastoma cells in serum-free, stem cell-selective medium is an effective method to dissociate and purify tumor stem cells in vitro.
\end{abstract}

Key words: Tumor spheres, tumor stem cell, neuroblastoma

Neuroblastoma, a neuroendrocrine tumor arising from any neural crest element of the sympathetic nervous system, accounts for eight to ten percent of all solid tumors in children. Advances in chemotherapeutic regimen and complex treatment have improved the prognosis of many types of pediatric cancer. Starkly however, the prognosis of

\footnotetext{
Correspondence to: Ben-Shang Li; Tel: +86-13816009925;

Email: leebenshang@hotmail.com

This paper was translated from Chinese into English by CJC Medical Translation and edited by Wei Liu.

Received: 2010-06-24; Accepted: 2010-08-19

Grants: Science and Technology Commission of Shanghai City (No. 08411953700); Research Foundation from Health Bureau of Shanghai City (No. 2008026)
}

neuroblastoma has not gained significant progress, especially the rates of metastasis for advanced patients, recurrence and long term survival $(<40 \%)^{[1]}$.

A fraction of cancerous cells, termed cancer stem cells, demonstrate proliferative and pluripotent capacity and determine the initiation and development of cancer ${ }^{[2]}$. In addition, cancer stem cells are correlated with tumorigenesis, metastasis, recurrence and drug resistance. Support for the cancer stem cell hypothesis has been demonstrated by studies involving tumorigenic surface marker expression. CD34 /CD38 cancer stem cells have been found in human acute monocytic leukemia ${ }^{[3]}$ and CD44 $/$ CD24- cancer stem cells have been isolated from breast cancer ${ }^{[4]}$. Subsequently, cancer stem cells have been identified in numerous solid cancers. The existence of 


\section{Chinese Journal of Cancer}

cancer stem cells may be the main reason for poor prognosis of advanced neuroblastoma. As cancer stem cells account for less than one percent of the total cancer cell population, their isolation and purification is a major obstacle to be overcome in an effort to further elucidate cancer stem cell biology.

Cells endowed with the properties of self-renewal and proliferation form floating spherical structures when growing in serum-free medium supplemented with epidermal growth factor (EGF) and basic fibroblast growth factor (bFGF). These spheres enrich stem cells and progenitor cells, facilitating differentiation into three dimensional structures ${ }^{[5]}$. Single cells isolated from tumor spheres can proliferate and form spheres in serum-free medium, whereas differentiated cells expire. In vitro sphere formation and serial passage are considered hallmarks of stem cells and progenitor cells. Therefore, cancer stem cells can be cultured to homogeneity in specific, serum-free medium.

The method of in vitro sphere formation was initially employed for the isolation and analysis of mammalian nervous system and breast stem cell tissue. In succeeding studies, stem cells and progenitor cells originating from cancerous tissue were found to generate tumor spheres in vitro ${ }^{[6]}$. Neurospheres, initially isolated and amplified from human neuroglioma, maintain the properties of neural stem cells and inherit the morphology and genotype of primary neuroglioma ${ }^{[7]}$. In vitro sphere formation has been widely applied for isolating cancer stem cells from breast and brain cancer. However, the application of in vitro sphere formation has not been elucidated for neuroglioma. In the present study, neuroglioma cells were cultured in specific, serum-free medium, establishing a new method for the isolation and purification of neuroglioma cancer stem cells.

\section{Materials and Methods}

\section{Materials}

DMEM/F12, B27, type I collagenase and 0.25\% trypsin (GIBCO, USA), EGF and bFGF (Invitrogen, USA), 13-cis retinoic acid (Sigma, USA), reverse transcription kit (Promega, USA), and Ex-Taq PCR kit (Takara Bio, Japan) were purchased. Nude mice (4-6 weeks old) were purchased from SLRC laboratory animal center (Shanghai, China); the license number was SCXK (Hu) 2007-0005.

\section{Methods}

\section{Tumor sphere culture and differentiation}

Three milliliters of bone marrow aspirate were obtained from pediatric patients diagnosed with stage IV neuroblastoma. Single cells were dissociated by Ficoll and cultured in serum-free DMEM/F12 medium supplemented with $20 \mu \mathrm{mol} / \mathrm{L}$ EGF, $20 \mu \mathrm{mol} / \mathrm{L}$ bFGF and 2\% B27 in 25 $\mathrm{cm}^{2}$ flasks, plated at a density of $1 \times 10^{5} \mathrm{cells} / \mathrm{mL}$ and incubated at $37^{\circ} \mathrm{C}$ in a humidified $5 \% \mathrm{CO}_{2}$ incubator. Fresh medium was added to the cells every three days. Cells were passaged at the ratio of $1: 3$ every seven days.

Tumor spheres were collected by filtering, dissociated into single cells and cultured in DMEM/F12 supplemented with $10 \%$ FBS. After cells were adherent, $5 \mu \mathrm{mol} / \mathrm{L}$ 13-cis retinoic acid (diluted in DMSO) was added to cells to induce cell differentiation. Cells were passaged by trypsinization every three days and 13-cis retinoic acid was added.

\section{Single cell tumor sphere formation assay}

Tumor spheres were dissociated into a single cell suspension at the concentration of $1 \times 10^{3}$ cells $/ \mathrm{mL}$. Cells were diluted 100 times to form a 10 cells $/ \mathrm{mL}$ suspension in $20 \mathrm{~mL}$ of serum-free medium. $100 \mu \mathrm{L}$ of suspended cells were seeded, in triplicate, into each well of a 96 well plate. Single cells were marked and observed every day. The cloning formation rate and single cell tumor sphere formation rate was calculated after 7 days.

\section{$R T-P C R$}

RNA was extracted from primary neuroblastoma cells and sphere-forming cells; cDNA was synthesized according to manufacturer's instructions (Promega). The expression of stem cell markers Bmi-1 and Oct- 4 was detected by RT-PCR, with GAPDH serving as a negative control. The primers for Bmi-1 were 5'-GTGTGTGCTTTGTGGAG-3' and 5'-TGGTCTGGTCTTGTGAAC-3'; the product was 149 base pairs. The primers for Oct-4 were 5'-GACAACAATGAAAATCTTCAGGAG -3' and 5'-TTCTGGCGCCGGTTACAGAACCA- 3'; the product was 218 base pairs. The primers for gapdh were 5'-GGACCTGACCTGCCGTCTAG3 ' and 5'-TAGCCCAGGATGCCCTTGAG-3'; the product was 99 base pairs.

The PCR system contained $1 \mu \mathrm{L}$ cDNA, $0.125 \mu \mathrm{L}$ Ex-Taq, $2 \mu \mathrm{L}$ Taq buffer, $2 \mu \mathrm{L}$ dNTP mixture, $1 \mu \mathrm{L}$ forward primer, $1 \mu \mathrm{L}$ reverse primer and $12.875 \mu \mathrm{L}$ water with cycling as follows: pre-denaturalized at $94^{\circ} \mathrm{C}$ for $5 \mathrm{~min}$, denaturalized at $94^{\circ} \mathrm{C}$ for $30 \mathrm{~s}$, annealed at $50^{\circ} \mathrm{C}$ (Oct-4) $152^{\circ} \mathrm{C}(\mathrm{Bmi}-1) / 56^{\circ} \mathrm{C}(\mathrm{GAPDH})$ for $30 \mathrm{~s}$ and extended at $72^{\circ} \mathrm{C}$ for $30 \mathrm{~s}$ (30 cycles) and followed by a extension at $72^{\circ} \mathrm{C}$ for 2 min.

\section{Xenograft formation}

Four to six week old nude mice were maintained and treated under pathogen-free specific conditions. Generation two and three neurospheres were dissociated into single cell suspensions and cell concentrations were adjusted to $2 \times$ $10^{7} / \mathrm{mL}, 2 \times 10^{5} / \mathrm{mL}, 2 \times 10^{4} / \mathrm{mL}$ or $2 \times 10^{3} / \mathrm{mL}$. Adjusted cells were diluted with phosphate buffered saline (PBS) at a $1: 1$ ratio and were subcutaneously injected into the armpit of nude mice $\left(1 \times 10^{6}, 1 \times 10^{4}, 1 \times 10^{3}\right.$ or $1 \times 10^{2}$ cells; respectively). Each serial dilution was implanted in triplicate 


\section{Chinese Journal of Cancer}

mice. Tumor xenograft formation was observed twice every week. Tumor xenografted mice were sacrificed by cervical dislocation and fixed in $75 \%$ ethanol for $15 \mathrm{~min}$. Xenograft was collected and immersed in methanol or cut into $1 \mathrm{~cm}^{2}$ pieces and digested in $0.15 \%$ type I collagenase at $37^{\circ} \mathrm{C}$. Upon forming a single cell suspension (8-10 h), cells were rinsed with PBS three times and cultured as previously described. Cells were re-injected into nude mice 3 weeks later.

\section{Results}

\section{Tumor sphere culturing}

Floating tumor spheres were formed within 4 to 6 days of culturing in serum-free medium (Figure 1A). After 7 days, cells were passaged, forming new tumor spheres. As shown in Figure 1B, spheres were adherent and differentiated after culturing in medium containing $10 \%$ FBS.

\section{Single cell tumor sphere formation in vitro}

Employing a single cell tumor-sphere formation assay, three types of cell morphology were observed. Type 1: forming classic single tumor sphere; type 2: cells were adherent and distributed diffusely; type 3: forming multi-tumor spheres. The average formation rate of tumor spheres was $55.3 \%$ (types 1-3). The single cell tumor sphere formation rate was $26.3 \%$ (types 1 and 3 ), indicating spheres display different states of division and proliferation, attributed to different states of differentiation. $44.7 \%$ of cells were totally differentiated, verified by a lack of proliferation under serum-free conditions. $29 \%$ of cells were relatively differentiated and proliferative, but distributed diffusely or were adherent and did not form spheres. Cells with stem cell characteristics accounted for $26.3 \%$, generating floating spheres which ultimately formed multi-tumor spheres (Figure 2).

\section{Expression of Oct-4 and Bmi-1}

As demonstrated by RT-PCR, stem cell expression of Oct-4 and Bmi-1 was elevated in sphere-forming cells relative to primary tumor cells, suggesting that tumor spheres enriched cancer stem cells (Figure 3).

\section{Xenograft formation}

14 days post-injection, nude mice injected with $1 \times 10^{6}$ (3 of 3 ) or $1 \times 10^{4}$ (2 of 3 ) sphere-forming cells formed xenografts; those injected with $1 \times 10^{3}$ or $1 \times 10^{2}$ sphereforming cells did not. In contrast, xenograft formation was reserved for nude mice injected with $n \times 10^{8}$ or $n \times 10^{6}$ neuroblastoma cells. Our results indicate tumor spheres possess a higher capacity toward xenograft formation. Serial transplantation assay demonstrated the rate of tumor formation at $75 \%$ for the second generation and $100 \%$ for the third generation, indicating sphere-forming cells continuously form xenografts. Immunohistochemical analysis verified xenograft expression of neuroblastoma specific antigen Chromogranin $\mathrm{A}(\mathrm{CgA})$ and neuron-specific enolase (NSE) (Figure 4).

\section{3-cis retinoic acid induces tumor sphere cell differentiation}

Prior to induction, sphere-forming cells visibly demonstrated triangular or star-like morphology. Following treatment, cell protrusions increased and elongated, differentiating into a neuronal morphology (Figure 5).
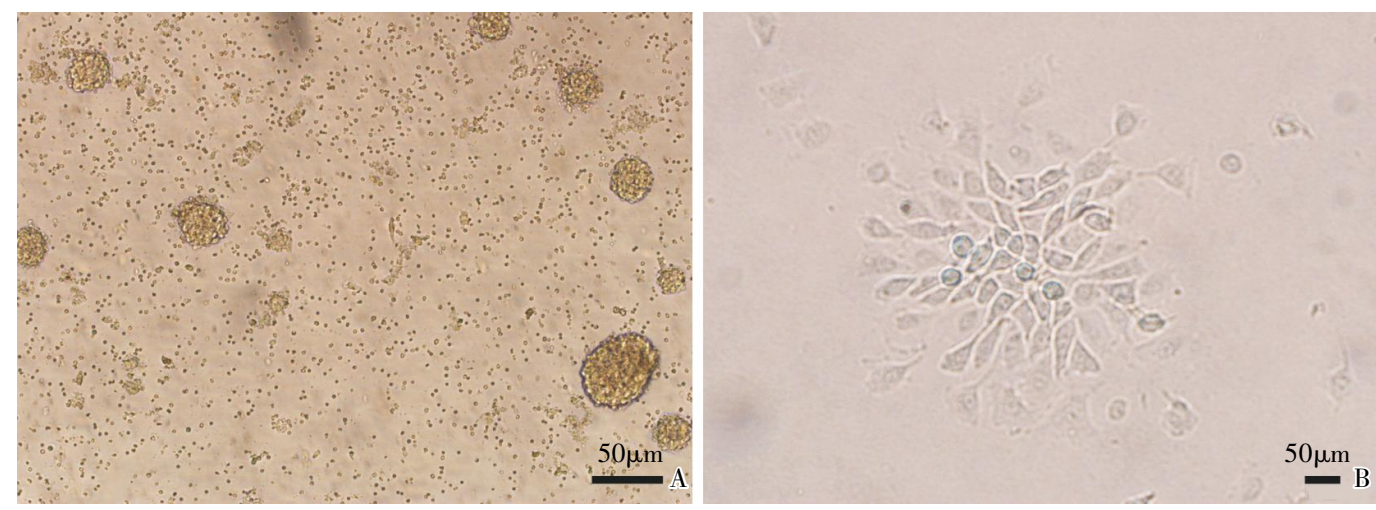

Figure 1 Tumor sphere formation

A, bone marrow derived neuroblastoma cells form nonadherent spheres under serum-free stem cell conditions after incubation for 4 to 6 days; B, tumor sphere cells attach to the culture dish and tend to differentiate when cultured in medium containing $10 \%$ FBS. 


\section{Chinese Journal of Cancer}

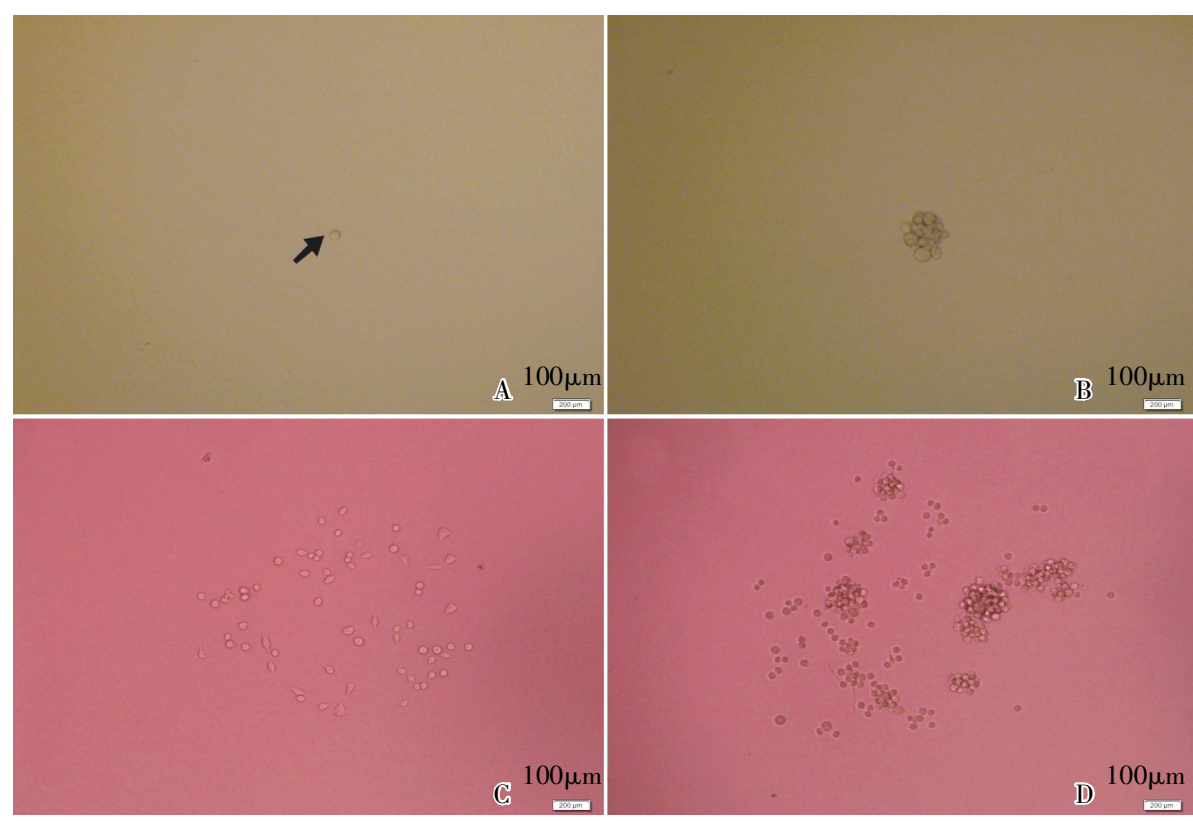

Figure 2 Clonal analysis of single sphere-forming cells

Cells were tumor sphere derived and cultured in serum-free, stem cell medium simultaneously.

A, a single sphere-forming cell (indicated by arrow); B, a reforming tumor sphere from a single sphere-forming cell (5 days post); $\mathrm{C}$, relatively differentiated cells attach to the culture dish and do not form tumor spheres; D, cells possessing stem cell characteristics reform tumor spheres (7 days post).

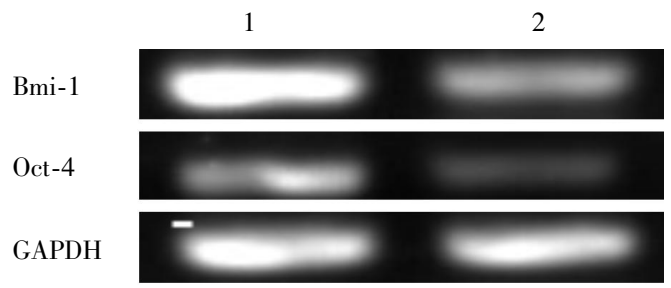

Figure 3 Expression of Bmi-1 and Oct-4 in sphere-forming cells and primary tumor cells

Lane 1, sphere-forming cells cultured in serum-free, stem cell medium for 2 weeks; lane 2, primary tumor cells. GAPDH serves as control.

\section{Discussion}

Despite the acceptance of the cancer stem cell hypothesis, little progress has been made toward understanding the biological characteristics of cancer stem cells, principally due to technical difficulties in their isolation and purification. Several methods have been employed for isolation and identification of cancer stem cells, including side population (SP) and ALDEFLUOR assays. However, these methods are complex and expensive ${ }^{[8]}$. The simplicity and convenience of the in vitro single cell tumor sphere formation assay has been successfully applied to the isolation of many types of cancer stem cells.
In the present study, floating spheres formed from primary neuroblastoma cells after 4 to 6 days of culturing in serum-free medium supplemented with EGF and bFGF. Moreover, sphere-forming cells survived continuous passage in culture. In contrast, tumor cells which did not proliferate under serum-free conditions expired during serial passage. Our results reveal a fraction of primary neuroblastoma tumor cells can proliferate without serum.

Similar to stem cells, cancer stem cells are proliferative and differentiate ${ }^{[8]}$. Floating spheres remained stable under repeated passage in culture. Also, sphere-forming cells formed xenografts in nude mice. The rates of xenograft formation of the second generation and third generation were $75 \%$ and $100 \%$ respectively, indicating a fraction of 


\section{Chinese Journal of Cancer}
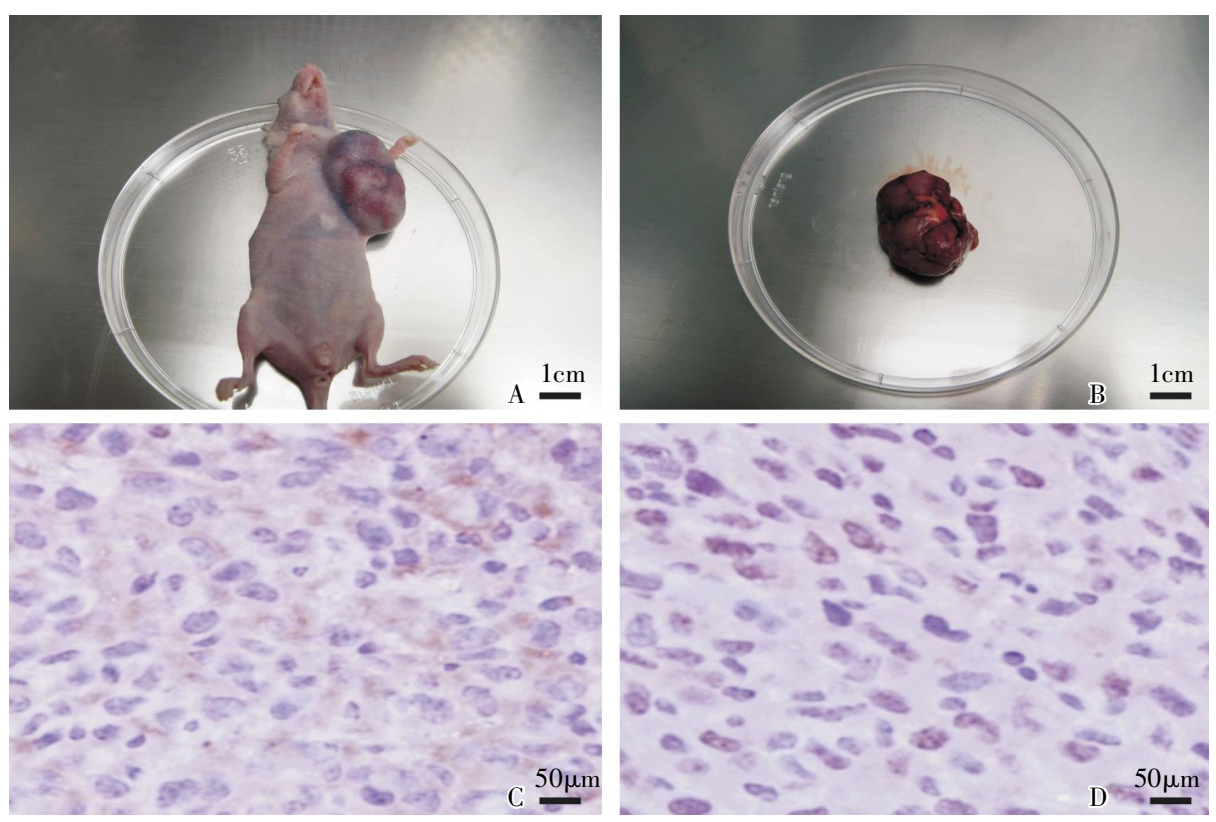

Figure 4 Sphere-forming cell propogation in nude mice

A total of $1 \times 10^{4}$ sphere-forming cells were dissociated and injected into the armpit of nude mice; tumor mass was observed 14 days later. A and B, xenograft tumor growth 30 days post implantation; $C$ and D, xenograft tumor cells are CgA- and NSE-positive as verified by specific peroxidase staining.
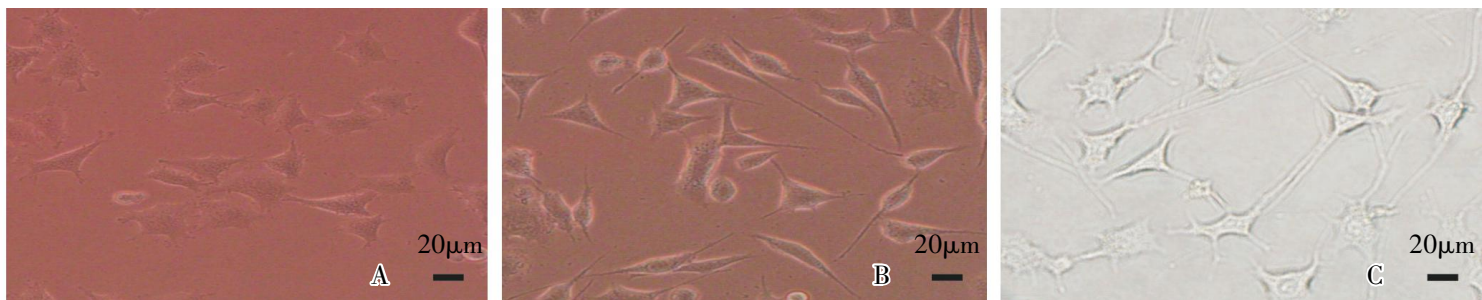

Figure 5 13-cis retinoic acid induces neruosphere-forming cell differentiation to neural cells

Sphere-forming cells cultured in differentiation medium supplemented with $5 \mathrm{M} \mathrm{13-cis} \mathrm{retinoic} \mathrm{acid} \mathrm{attached} \mathrm{to} \mathrm{the} \mathrm{bottom} \mathrm{of} \mathrm{the} \mathrm{culture} \mathrm{dish} \mathrm{and}$ protruded cell processes. Most cells exhibited neuronal morphology, extending processes and forming a network. Neurosphere cell morphology before (A), 3 days (B) and 9 days (C) after induction, respectively.

tumor cells possess self renewal and proliferation. Following treatment with 13-cis retinoic acid, cell protrusions increased, elongated and differentiated, displaying morphology indicative of a neuron. These results demonstrate tumor spheres have cells endowed with the specific properties of stem cells.

Oct-4 and Bmi-1 are important factors contributing to the proliferation and differentiation of stem cells, as well as maintaining stem cell character ${ }^{[9,10]}$. We showed elevated Oct-4 and Bmi-1 expression in sphere-forming cells relative to primary tumor cells, suggesting a sphere population contains a greater proportion of stem cells than in a primary tumor cell population.

A cell population includes stem cells, progenitor cells, rapid proliferated cells and differentiated cells. Multi-potent stem cells produce progenitor cells possessing a broad differentiation potential. Progenitor cells further differentiate into rapid proliferated cells, which finally generate differentiated cells. Like normal stem cells, the development of cancer stem cells displays a process of gradual differentiation [11]. Applying the single cell tumor sphere formation assay, we observed tumor spheres include several cell types. Well differentiated cells did not form single cell tumor spheres. Partially differentiated cells formed single cell spheres, but not tumor spheres. Undifferentiated stem cells and progenitor cells formed single cell tumor spheres $(26.3 \%)$ at a much higher percentage than primary tumor cells $(<1 \%)$.

Sphere-forming cells, endowed with the capacities of self renewal and the potential to differentiate, expressed 


\section{Chinese Journal of Cancer}

levels of stem cell markers and efficiently generated

xenografts in nude mice. These findings suggest tumor spheres accumulated cancer stem cells. Our data indicated culturing neuroblastoma in serum-free medium was an efficient method to isolate and purify cancer stem cells. The establishment of this method provided the foundation for further screening neuroblastoma stem cell markers and identifying the pathogenesis of neuroblastoma. In addition, our findings establish a new molecular diagnosis method and targeted therapy for neuroblastoma.

\section{References}

[1] Maris JM, Hogarty MD, Bagatell R, et al. Neuroblastoma [J] Lancet, 2007, 369(9579): 2106-2120.

[2] Pardal R, Clarke MF, Morrison SJ. Applying the principles of stemcell biology to cancer [J]. Nat Rev Cancer, 2003, 3(12): 895-902.

[3] Bonnet D, Dick JE. Human acute myeloid leukemia is organized as a hierarchy that originates from a primitive hematopoietic cell [J].
Nat Med, 1997, 3(7):730-737

[4] Al-Hajj M, Wicha MS, Benito HA, et al. Prospective identification of tumorigenic breast cancer cells [J]. Proc Natl Acad Sci, 2003, 100 (7): 3983-3988

[5] Tropepe V, Sibilia M, Ciruna BG, et al. Distinct neural stem cells proliferate in response to EGF and FGF in the developing mouse telencephalon [J]. Dev Biol, 1999, 208(1): 166-188.

[6] Nicolis SK. Cancer stem cells and "stemness" genes in neurooncology [J]. Neurobiol Dis, 2007, 25(2):217-229.

[7] Ignatova TN, Kukekov VG, Laywell ED, et al. Human cortical glial tumors contain neural stem-like cells expressing astroglial and neuronal markers in vitro [J]. Glia, 2002, 39(3):193-206.

[8] Boman BM, Wicha MS. Cancer stem cells: a step towards cure [J]. J Clin Oncol, 2008, 17(26): 2795-2799.

[9] Atlasi Y, Mowla SJ, Ziaee SA, et al. OCT-4, an embryonic stem cell marker, is highly expressed in bladder cancer [J]. Int $J$ Cancer, 2007, 120(7):1598-1602.

[10] Molofsky AV, Pardal R, Iwashita T, et al. Bmi-1 dependence distinguishes neural stem cell self-renewal from progenitor proliferation [J]. Nature, 2003, 425(6961): 962-967.

[11] Lobo N A., Shimono Y, Qian, D, et al. The biology of cancer stem cells [J]. Annu Rev Cell Dev Biol, 2007, 23:675-699. 